\title{
Tungsten Probe Tip Cleaning
}

Jehan Saujauddin $^{1}$, Tim Niemi ${ }^{1}$, Ted Lundquist ${ }^{1}$, Baohua Niu ${ }^{1}$ and Michael Cable ${ }^{2}$

${ }^{1}$ Carl Zeiss SMT Inc., Pleasanton, California, United States, ${ }^{2}$ XEI Scientific, Inc., Redwood City, California, United States

Nanoprobing has become indispensable for the characterization of FEOL processes and FinFET performance in early process development and HVM yield improvements [1-3]. When the processes and transistor performance are fully characterized such as transistor level I-V curves (performance) and leakage (power efficiency), the risk to process development is greatly reduced, providing high impact to the HVM-product performances.

For leading edge CMOS processes, transistor performance (Idsat and Ioff) is impacted by excessive electron energy and beam current, as nanoprobing uses a electron beam to image an area of interest and guide the landing of the nanometer-sized probe tip. Imaging is done at electron energies of $200 \mathrm{eV}$ and low beam current, typically $\sim 10$ to $30 \mathrm{pA}$ [1]. The probe tips and sample must remain clean before reliable electrical measurements can be made. Normally, plasma cleaning using air is employed to remove contamination from the sample and probes.

Electron beam induced hydro-carbon deposition has long been identified as the major source of probe tip contamination. Electron beam depositions, of hydro-carbons, which exist in the vacuum system or migrate into the vacuum system during sample exchange is the main problem. As carbon deposition occurs, conductivity degrades and ruins both the imaging resolution and the probe-to-sample conductive contacts. The carbon deposition is readily removed by plasma cleaning with air, but this oxidizes the probe tips, increasing their electrical resistance. The standard approach to remove tungsten is "cross cleaning". Two probe tips are set to touch each other (See Figure 1) and then an electrical current is passed between them. The current heats the tungsten to decompose the oxide and release oxygen. As nanoprobing continues, more carbon contamination is grown on the probes.

Oxygen removes carbon contamination, but oxidization increases the resistance between the probe tips and negatively impacts electrical measurements. Instead of being bright, the tip suggests it is insulating or at least a poor conductor. Oxidation of the probes during plasma cleaning with Air increased their resistance which degrades measurements.

Further, nanoprobing can be tedious due to the care needed to place a $10 \mathrm{~nm}$ diameter probe tip on sub 20 $\mathrm{nm}$ transistor contacts and doing this multiple times on the same contacts. It is imperative to have both clean and low resistance probe tips. At Zeiss PCS, we are exploring BKMs to streamline the nanoprobing process as well as the reliability and consistency of measurements. ZEISS PCS has investigated both sample and probe tip cleaning BKMs using existing and new plasma clean methods.

In a collaboration between Zeiss PCS and XEI, we experimented with $\mathrm{Ar}-\mathrm{H}_{2}$ mixtures instead of air for our plasma cleaning. This new plasma cleaning chemistry works very well (See Figure 2). It is now the standard configuration for Zeiss PCS SEM based probing tools. This new process does not replace crosscleaning of new tips, but it does remove the frequent build-up of contaminants on the probes, which means less down time due to cross-cleaning. 

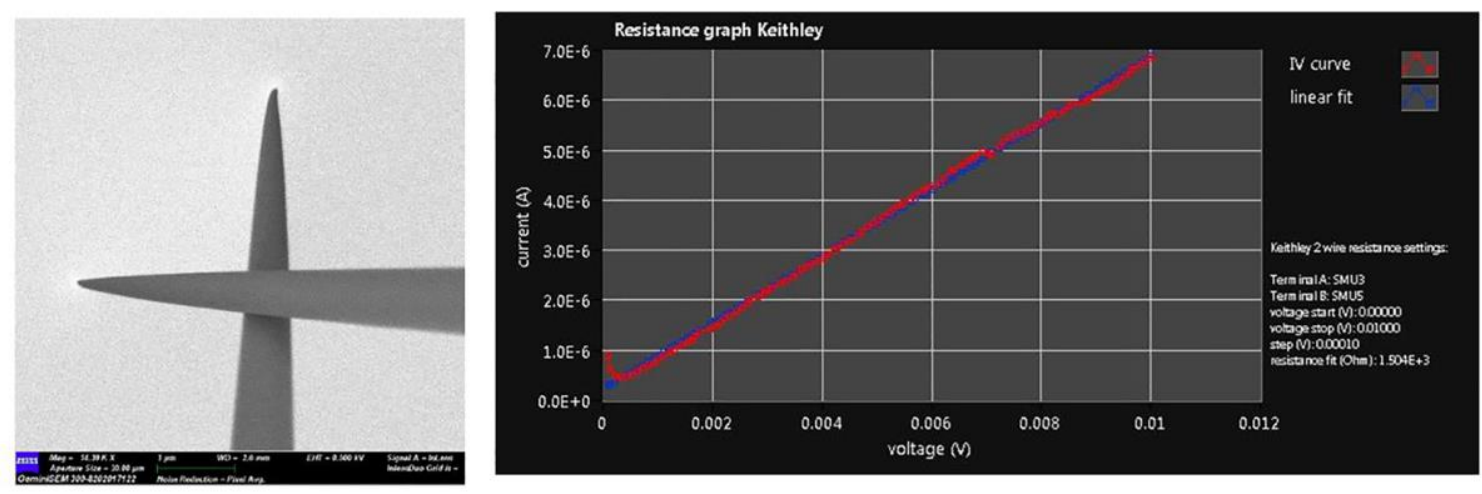

Figure 1. Left: Image of two probes set for cross cleaning reveals an oxide degradation effect on secondary electron emission. Right: Resistance between probe tips after oxygen plasma "cleaning".

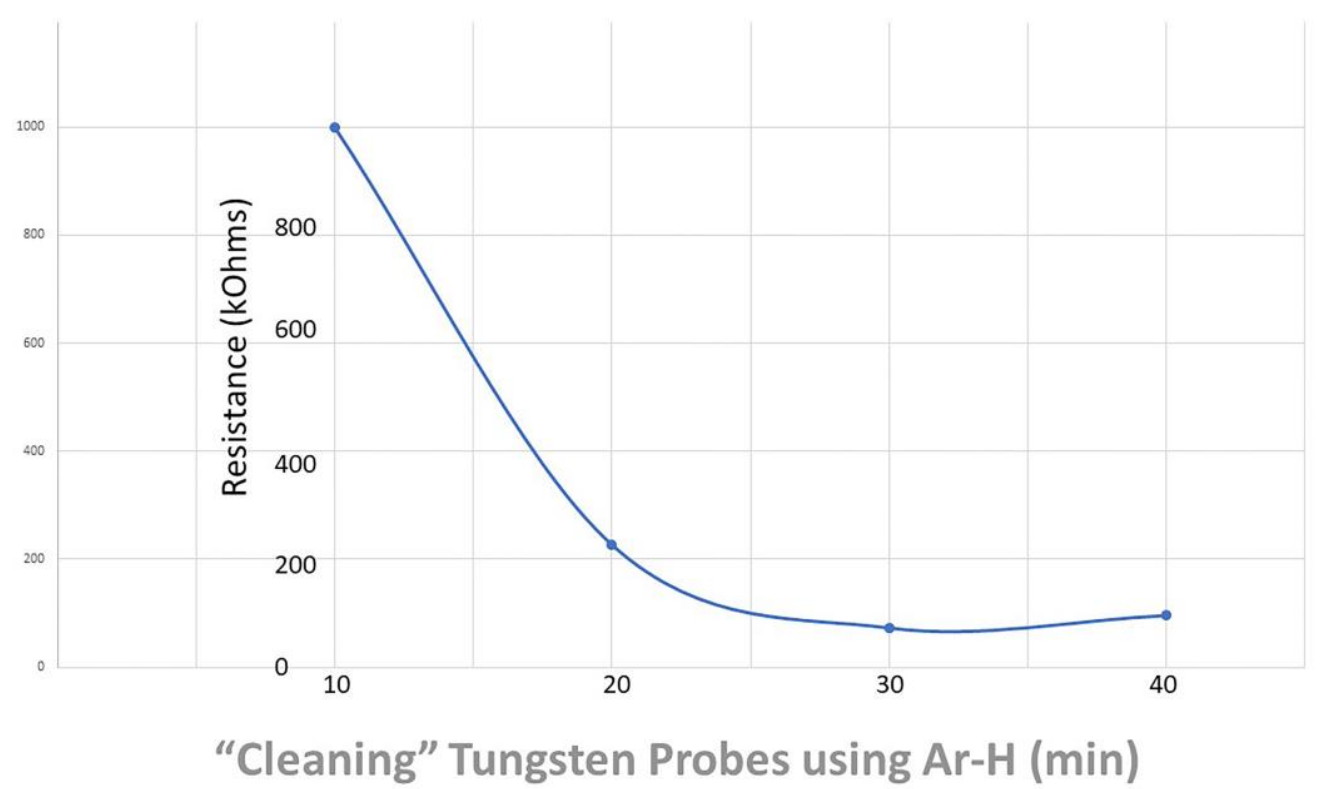

Figure 2. Decrease in resistance of tungsten probes cleaned with AR/H plasma at 35W.

\section{References}

[1] N. Jehan Saujauddin, E.P.Y. Chen, F Beaudoin, Nanoprobing Beyond 14nm with Latest Generation SEM-based Nano-probes, $43^{\text {rd }}$ ASM-ISTFA 2017 Proceedings.

[2] M. von Haartman, S. Rahman, S. Ganguly, J. Verma, A. Umair, T. Deborde, Optical Fault Isolation and Nanoprobing Techniques for the $10 \mathrm{~nm}$ Technology Node and Beyond, 41 ${ }^{\text {st }}$ ASM-ISTFA 2015 Proceedings.

[3] A Qiu, W Lowe, M Arora, Nanoprobing on $7 \mathrm{~nm}$ FinFET Devices in an SRAM Array: Challenges and Solutions, $45^{\text {th }}$ ASM-ISTFA 2019 Proceedings. 